\title{
Clemastine fumarate as a remyelinating therapy for multiple sclerosis (ReBUILD): a randomised, controlled, double-blind, crossover trial
}

\begin{abstract}
AriJ Green, Jeffrey M Gelfand, Bruce A Cree, Carolyn Bevan, WJohn Boscardin, Feng Mei, Justin Inman, Sam Arnow, Michael Devereux, Aya Abounasr, Hiroko Nobuta, Alyssa Zhu, Matt Friessen, Roy Gerona, Hans Christian von Büdingen, Roland G Henry, Stephen L Hauser, Jonah R Chan
\end{abstract}

\begin{abstract}
Summary
Background Multiple sclerosis is a degenerative inflammatory disease of the CNS characterised by immune-mediated destruction of myelin and progressive neuroaxonal loss. Myelin in the CNS is a specialised extension of the oligodendrocyte plasma membrane and clemastine fumarate can stimulate differentiation of oligodendrocyte precursor cells in vitro, in animal models, and in human cells. We aimed to analyse the efficacy and safety of clemastine fumarate as a treatment for patients with multiple sclerosis.
\end{abstract}

Methods We did this single-centre, 150-day, double-blind, randomised, placebo-controlled, crossover trial (ReBUILD) in patients with relapsing multiple sclerosis with chronic demyelinating optic neuropathy on stable immunomodulatory therapy. Patients who fulfilled international panel criteria for diagnosis with disease duration of less than 15 years were eligible. Patients were randomly assigned (1:1) via block randomisation using a random number generator to receive either clemastine fumarate $(5.36 \mathrm{mg}$ orally twice daily) for 90 days followed by placebo for 60 days (group 1 ), or placebo for 90 days followed by clemastine fumarate $(5.36 \mathrm{mg}$ orally twice daily) for 60 days (group 2). The primary outcome was shortening of P100 latency delay on full-field, pattern-reversal, visual-evoked potentials. We analysed by intention to treat. The trial is registered with ClinicalTrials.gov, number NCT02040298.

Findings Between Jan 1, 2014, and April 11, 2015, we randomly assigned 50 patients to group $1(\mathrm{n}=25)$ or group 2 $(n=25)$. All patients completed the study. The primary efficacy endpoint was met with clemastine fumarate treatment, which reduced the latency delay by $1.7 \mathrm{~ms} /$ eye $(95 \% \mathrm{CI} 0 \cdot 5-2 \cdot 9 ; \mathrm{p}=0.0048)$ when analysing the trial as a crossover. Clemastine fumarate treatment was associated with fatigue, but no serious adverse events were reported.

Interpretation To our knowledge, this is the first randomised controlled trial to document efficacy of a remyelinating drug for the treatment of chronic demyelinating injury in multiple sclerosis. Our findings suggest that myelin repair can be achieved even following prolonged damage.

Funding University of California, San Francisco and the Rachleff Family.

\section{Introduction}

Multiple sclerosis is an inflammatory, autoimmune demyelinating disease of the CNS in which an adaptive immune response targets unknown CNS antigens resulting in oligodendrocyte damage and neurological dysfunction. ${ }^{1.2}$ Myelin is a specialised extension of the plasma membrane of oligodendrocytes that ensheathes axons and enables saltatory conduction of action potentials. Patients with multiple sclerosis with myelin injury exhibit impaired conduction of action potentials, which can manifest as neurological dysfunction in the affected pathway. Only terminally differentiated oligodendrocytes can form myelin and enwrap CNS axons. ${ }^{3,4}$ Newly differentiated oligodendrocytes have the capacity to remyelinate denuded axons, and a shortterm crucial window for myelination might exist at the time of differentiation. ${ }^{5}$ Despite the presence of oligodendrocyte precursors in multiple sclerosis lesions, ${ }^{6-8}$ remyelination is generally unsuccessful —and at best partial-after demyelinating injury in multiple sclerosis. ${ }^{910}$
Current treatments for multiple sclerosis block access of immune cells to their target tissue or otherwise suppress inflammatory injury, but do not fully prevent neuroaxonal degeneration and disability.1.12 ${ }^{112}$ proven treatments are available to remyelinate or otherwise sustainably repair myelin-related injury.

Diagnosis of multiple sclerosis is dependent on evidence of dissemination of lesions in time and space, meaning that previous demyelinating injury must be present at the time of diagnosis. ${ }^{2.13}$ Even at disease onset, many patients show evidence of myelin damage beyond the clinically salient lesion that brought them to medical attention. Furthermore, myelin disruption in multiple sclerosis extends to the normal appearing white matter outside of circumscribed lesions, especially in the later stages of disease..$^{14}$ Chronic demyelination is believed to contribute to early axonal loss and resultant progressive disability. ${ }^{2,1516}$

Whether or not remyelination can be achieved in chronically demyelinated lesions in multiple sclerosis remains an unanswered question. A binary, cell-specific,
Published Online October 10, 2017 http://dx.doi.org/10.1016/ S0140-6736(17)32346-2 See Online/Comment http://dx.doi.org/10.1016/ S0140-6736(17)32639-9

Department of Neurology (A) Green MD, J M Gelfand MD, B A Cree MD, C Bevan MD, F Mei PhD, J Inman BS, S Arnow BS, M Devereux BS, A Abounasr BS, A Zhu BS, $H C$ von Büdingen MD, Prof R G Henry PhD, Prof S L Hauser MD, Prof J R Chan PhD), Department of Ophthalmology (AJ Green),

Department of Epidemiology and Biostatistics

(Prof WJ Boscardin PhD), Program in Neuroscience (F Mei, Prof S L Hauser, ProfJ R (han), Department of Obstetrics and Gynecology (M Friessen PhD, Prof R Gerona PhD), Department of Radiology and Biomedical Imaging (Prof R G Henry), and Department of Pediatrics and Neurosurgery and Eli and Edythe Broad Institute for Stem Cell Research and Regeneration Medicine, University of California, San Francisco, San Francisco, CA, USA (H Nobuta PhD); and Bioengineering Graduate Group, University of California, Berkeley, and San Francisco, San Francisco, CA, USA (Prof R G Henry)

Correspondence to: Dr AriJ Green, Department of Neurology and Department of Ophthalmology, Sandler Neurosciences Center, University of California San Francisco, San Francisco, CA 94158, USA agreen@ucsf.edu 
Research in context

\section{Evidence before this study}

We searched PubMed articles published until Jan 10, 2017, without language restrictions reporting on remyelinating trials and treatments for multiple sclerosis. In our search, we used the terms "multiple sclerosis" OR "MS" OR "optic neuropathy" OR "visual evoked potential" OR "clemastine" AND "remyelination" initially filtering results for articles that report phase 1, 2, or 3 clinical trials. In addition, abstracts were reviewed from the American Academy of Neurology Meeting (AAN) and European Committee on Treatment in Multiple Sclerosis between 2006 and 2016. No previously successful randomised controlled trials using a remyelinating drug to treat a chronic demyelinating injury were reported. A number of clinical trials for acute relapses that principally focused on optic neuritis were identified, including one that assessed visual-evoked potentials (VEPs) as a secondary endpoint in a trial comparing simvastatin with placebo. This trial reported positive results in an intention-to-treat analysis but mismatch in severity was significant between the two groups, with the placebo group having more severe injury at baseline than the treated group. A successful phase 2 clinical trial of intravenous erythropoietin displayed improved VEP latency at 4 months as a secondary outcome in the treated group. In addition, a trial (RENEW) of opicinumab, a monoclonal antibody to inhibit LINGO 1, failed on its primary outcome in an intention-to-treat analysis, but showed apparent benefit in a per-protocol analysis. A second phase $2 b$ study (SYNERGY) of opicinumab in patients with relapsing and secondary progressive multiple sclerosis did not reach its primary endpoint: a multicomponent measure that evaluated improvement of physical function, cognitive function, and disability. An additional trial of intravenous immunoglobulin did not show a beneficial effect for VEP. A clinical trial that showed efficacy for neuroprotection with phenytoin in acute optic neuritis using optical coherence tomography peripapillary nerve fibre as an outcome did not show effects on VEP latency. In addition, two observational studies that monitored patients with multiple sclerosis showed that patients had worsening or stable P100 latency on VEP rather than spontaneous improvement, and one concluded that this would therefore make a good biomarker for measurement in remyelinating clinical trials. Additional observational and animal studies suggested that VEPs showed promise as putative measures of both demyelination and remyelination in multiple sclerosis and animal models of demyelinating injury.

\section{Added value of this study}

To our knowledge, this is the first clinical trial to establish the safety and efficacy of clemastine fumarate in patients with multiple sclerosis and to show a benefit from clemastine fumarate on VEP latency. Furthermore, to our knowledge, it is also the first successful trial to use a drug with substantial preclinical evidence to suggest a remyelinating effect that met its clinical endpoint with an intention-to-treat analysis and to show the effect of remyelinating therapy on chronic demyelination. Treatments capable of remyelination, neuroprotection, or regeneration are a major unmet need for multiple sclerosis and other diseases that involve myelin damage, loss, or dysfunction in the CNS. In the ReBUILD study, electrophysiological evidence of remyelination is seen in the context of a therapy with strong preclinical data, which suggests potential human efficacy. Furthermore, there is preliminary evidence to suggest that this effect might extend to low-contrast letter acuity-a well validated outcome of clinically relevant visual function in patients with multiple sclerosis.

\section{Implications of all available evidence}

Our results lend support to the further investigation of the safety and efficacy of clemastine fumarate in patients with acute demyelinating injury and other forms of chronic demyelination. They also provide evidence that supports further investigation to optimise timing and dosing of clemastine fumarate, and to assess its effects in other pathways of the CNS. Furthermore, this study suggests that the visual system is an attractive model for studying repair and remyelination in the setting of chronic demyelination. functional screening method identified clemastine

fumarate-a first generation antihistamine-as capable of inducing oligodendrocyte differentiation and myelination due to off-target antimuscarinic effects ${ }^{17}$ and was confirmed in a second independent screen.18 Clemastine fumarate readily crosses the blood-brain barrier and has been available over the counter in the USA since 1992. Preclinical work validated the efficacy of clemastine fumarate in vitro and in animal models..$^{1719}$ Additional work confirmed the efficacy of clemastine fumarate in multiple animal models ${ }^{17-22}$ and showed that this benefit was mediated specifically via remyelination induced from oligodendrocyte differentiation and not via effects on the immune system. ${ }^{19}$ We furthermore showed the capacity of clemastine fumarate to induce oligodendrocyte progenitor cell (OPC) differentiation and myelination with human OPCs (appendix).

Evoked potentials record cortical responses to a repetitive stimulus and can measure the speed of conduction in the CNS. Myelinated axons conduct electrical signals at 70-100 times the speed of unmyelinated axons of the same diameter. Pattern-reversal visual-evoked potentials (VEPs) record cortical responses on the scalp overlying the occipital lobe in response to an

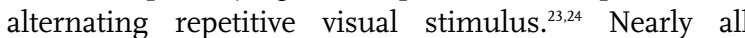
patients with multiple sclerosis ultimately exhibit demyelinating damage to the anterior visual pathway ${ }^{25}$ and detection of prolongation of VEP latency has been used as supportive evidence to help confirm a clinical diagnosis of multiple sclerosis. ${ }^{24,26}$ 
We did a phase 2 clinical trial to assess the efficacy of clemastine fumarate for remyelination in a state of chronic demyelination, evaluate the safety and tolerability profile for clemastine fumarate in patients with multiple sclerosis, and use a small molecule with well validated remyelinating potential to assess the responsiveness of putative, yet not validated, outcomes intended to measure remyelination.

\section{Methods}

\section{Study design and participants}

Before the study, preclinical work was done, which included generation of induced pluripotent stem cells (iPSCs) and culture and OPC differentiation from iPSCs (appendix). We did this double-blind, randomised, placebo-controlled, crossover trial (ReBUILD) at the University of California, San Francisco (San Francisco, CA, USA). Clinically stable patients with relapsing multiple sclerosis who fulfilled international panel criteria for diagnosis ${ }^{13}$ with disease duration of less than 15 years were eligible for screening. Although a previous clinically evident optic neuritis was not a requirement for enrolment, patients could not have had a clinical optic neuritis in the 6 months before screening or a documented optic neuritis in the qualifying eye for more than 5 years before screening. We designed inclusion criteria to ensure that patients had demyelinating injury in the visual pathway (VEP P100 latency in at least one eye of $118 \mathrm{~ms}$ ) and to increase the likelihood that the number of surviving axons was sufficient to provide the needed substrate for remyelination to occur (approximated by retinal nerve fibre layer thickness on spectral-domain optical coherence tomography [OCT] $>70 \mu \mathrm{m}$ in the VEP qualifying eye). Exclusion criteria included confounding ophthalmological disease that could affect vision or testing, changes in immunomodulatory therapy for multiple sclerosis in the 6 months before being randomly assigned, glucocorticoid use within 30 days before screening, concurrent use of 4-aminopyridine or fampridine, ${ }^{27}$ or serological evidence of vitamin B12 deficiency or hypothyroidism.

A Food and Drug Administration Investigational New Drug exemption was granted (on Oct 31, 2013; reference number 3398780 ) to investigate clemastine fumarate as a remyelinating medication for the treatment of multiple sclerosis. The study was approved by the UCSF Institutional Review Board and all participants provided informed consent. The trial was registered at ClinicalTrials. gov (number NCT02040298) before initiation of patient enrolment.

\section{Randomisation and masking}

We randomly assigned patients to either group 1 (active treatment over the first 90 days followed by placebo for 60 days) or group 2 (placebo for 90 days, followed by active treatment for 60 days) without a washout between the two periods. The differing length of the two epochs was intended to help determine if any difference in efficacy was based upon variation in exposure time.
The University of California, San Francisco (UCSF; San Francisco, CA, USA) investigational pharmacy randomly assigned (1:1) the patients (via block randomisation using a random number generator), and all patients and investigators were masked to group assignment for the duration of the trial, including the evaluation of all data and outcomes such as determination of VEP quality and final assignment of P100 latencies. Patients were reminded at each visit not to take any supplementary clemastine fumarate (or Tavist) and were asked if they had taken any added clemastine fumarate (no patients replied "yes").

\section{Procedures}

Medication was provided as unmarked blue capsules of $5.36 \mathrm{mg}$ clemastine fumarate (Teva Pharmaceuticals USA, Sellersburg, PA, USA) or placebo (corn starch; Medisca, Plattsburgh, NY, USA) by the UCSF investigational pharmacy. When on the active treatment, patients received $5.36 \mathrm{mg}$ orally twice daily $(10.72 \mathrm{mg} /$ day $)$.

We recorded monocular transient full-field patternreversal VEPs with check size 64-min of arc with a Nihon-Kohden MEB-2300 (Nihon-Kohden, Irvine, CA, USA). At least 100 averages were obtained per recording. VEPs were done at screening, baseline, and months 1, 3, and 5. VEP latency was defined by the P100. We averaged recordings from baseline and screening for comparison with final outcomes. We analysed VEP recordings for quality before unmasking and those recordings of insufficient quality to unequivocally identify the P100 were discarded before analysis. Investigators not involved in the clinical assessment of patients or acquisition of data assessed VEP quality and marking of P100 latency and were masked to any assignment information in batch at completion of the study.

For MRI analysis, all participants were scanned using a Siemens 3T Skyra scanner (Siemens, Erlangen, Germany) equipped with a 20-channel head-neck coil and a 32-channel spine coil array at baseline, and months 3 and 5. Sequences included myelin water fraction (MWF), magnetisation transfer ratio (MTR), and diffusion MRI.

To evaluate visual function, we assessed high-contrast and low-contrast visual acuity monocularly with retro-illuminated $100 \%$ and $2 \cdot 5 \%$ Sloan low-contrast vision charts, respectively (Precision Vision, La Salle, IL, USA). We recorded the number of letters the patients reported correctly and used this number for analysis. ${ }^{28}$

Evaluating clinicians masked to all other assessments did standardised clinical assessments, including the Expanded Disability Status Scale (EDSS), timed 25-foot walk (T25FW), and 6-min walk test (6MWT).

OCT (Spectralis; Heidelberg Engineering, Heidelberg, Germany) of the peripapillary retinal nerve fibre layer (RNFL) was done using a high-resolution ring B-scan $3 \mathrm{~mm}$ around the disc (target ART 10 and signal strength 25). 


\section{Outcomes}

The primary outcome was shortening of P100 latency delay on full-field, pattern-reversal VEPs. With the following ranking, based on the presumed relative importance of these secondary endpoints, whole brain MTR, white matter MTR, white matter fractional anisotropy (FA), and MWF were secondary imaging endpoints for efficacy. Additional assessments included standard T1 (both before and after administration of gadolinium) and T2 at all timepoints (appendix). The principal functional secondary endpoint was low-contrast letter acuity (LCLA). We also assessed change in RNFL thickness from baseline as an exploratory endpoint at months 3 and 5 .

Adverse events were recorded at each visit. We obtained safety laboratory measures including transaminases, triglycerides, creatinine, and thyroid-stimulating hormone concentrations at each visit. We assessed cognition with the symbol digit modality test (SDMT) that is sensitive to both cognitive dysfunction in multiple sclerosis $^{29}$ and cognitive dysfunction associated with excessive anticholinergic treatment in the elderly. ${ }^{30}$ We assessed fatigue via the multidimensional assessment of fatigue (MAF) scale. ${ }^{31,32}$

\section{Statistical analysis}

We did a power analysis before starting the trial, modelled on the parallel group portion of the trial looking at the 3-month outcome. We estimated that the sample size of 25 patients per group would give $90 \%$ power to detect a $50 \%$ relative reduction in latency with clemastine

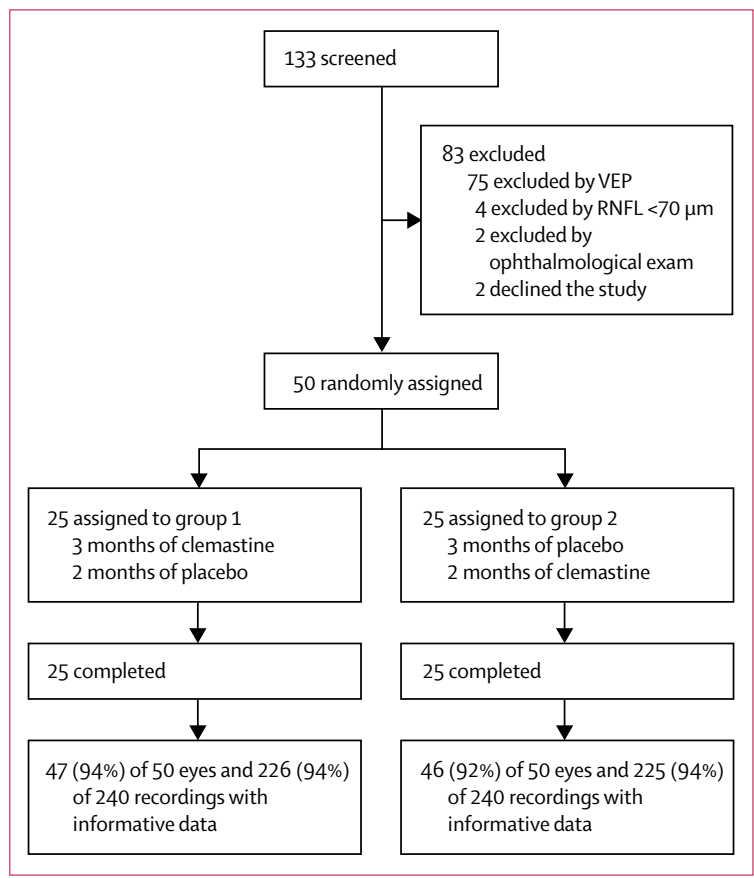

Figure 1: Trial design

VEP=visual-evoked potential. RNFL=retinal nerve fibre layer. fumarate compared with placebo at the 3-month timepoint. We analysed data with both a prespecified analysis plan appropriate for a repeated-measures crossover trial and by evaluating the trial post hoc as a delayed-treatment trial. We considered $\mathrm{p}$ values less than 0.05 to be significant. We used an intention-to-treat approach including all patients randomly assigned to the study. Missing outcome data were accounted for using mixed-effects linear models. For the principal analysis, we analysed the 30 day, 90 day (epoch 1), and 150 day (epoch 2) bivariate (left and right eye) measurements using mixed-effects linear regression. The crossover model included random effects for patient and for eyes within patient, and fixed-effects for the standard analysis of a $2 \times 2$ crossover model (an indicator for epoch 2 and an indicator for the active-treatment period) with additional adjustment for the baseline value. To mitigate the carryover effect and to optimally estimate the magnitude of the therapeutic effect, we did a parallel group comparison considering group 2 as subject to delayed treatment. In our primary analytical approach per the crossover analysis, the clemastine fumarate first group (group 1) was considered to be in the treatment group at months 1 and 3, and in the control group at month 5 , whereas the placebo first group (group 2) was considered to be in the control group at months 1 and 3 and in the treatment group at month 5 (and both groups were control group at baseline). This crossover analysis regards the effect of clemastine fumarate as transitory, lasting only while the active drug is being given, whereas the post-hoc delayed-treatment analysis regards each group as having a before and after clemastine fumarate period, with the assumption that the effect of the drug is persistent throughout the timeframe of the study. We also did an additional post-hoc analysis evaluating the frequency of patients who showed significant evidence of VEP latency improvement from baseline, which was defined as more than $6 \mathrm{~ms}$ of improvement consistent with laboratory standards and previous scientific literature. This analysis was done before review of the data, but was not initially specified in our protocol. Additional sensitivity analyses excluded the 30-day measurement as a repeated measurement in period 1 (90 days), as well as modelling the time change linearly to account for the differential exposure to treatment in period 1 and period 2 (60 days; appendix). We compared the means of continuous variables with $t$ tests and frequencies of categorical variables with $\chi^{2}$ tests. We averaged the values for variables with both screening and baseline values for subsequent comparison. An independent faculty analyst (WJB) did all analyses with Stata version 13

\section{Role of the funding source}

The funders of the study had no role in study design, data collection, data analysis, data interpretation, or writing of the report. The corresponding author had full 
access to all the data in the study and, along with JRC, had final responsibility for the decision to submit for publication.

\section{Results}

Between Jan 1, 2014; and April 11, 2015, we randomly assigned 50 patients to group $1(n=25)$ and group $2(n=25$; figure 1). The enrolled patients had an average age of $40 \cdot 1$ years (SD 10.3), mean mild disability with EDSS $2 \cdot 2(1 \cdot 1)$, and mean disease duration of $5 \cdot 1$ years $(5 \cdot 1$; table). Eyes with baseline RNFL thickness smaller than $70 \mu \mathrm{m}$ in the non-qualifying eye were excluded as prespecified. Furthermore, VEPs for which a reproducible P100 was not identified were excluded from analysis (constituting 29 [6\%] of 480 recordings), and therefore, $94 \%$ of all VEP recordings were included. 93 eyes were informative for the final trial outcomes after considering these exclusions. All patients who were randomly assigned attended all visits and completed the trial (figure 1).

28 patients $(56 \%)$ had a previous history of optic neuritis at an average of 4.3 years before enrolment (table). Baseline characteristics in terms of disability, VEP P100 latency, OCT measures, and other functional assessments were similar between the groups (table).

No clinical relapses occurred during the trial. 46 (92\%) of the 50 patients were on immunomodulatory disease modifying therapy: 20 on injectable, 16 on oral, and ten on high-potency infusible therapies. No patient had a change in immunomodulatory therapy during the course of the trial. No interaction for any of the therapies was seen on any of the outcomes, but the trial was not powered to detect such interactions.

Patients in both groups exhibited shortening of P100 latency while on the active compound. The primary prespecified efficacy endpoint for the trial was met with reduction of latency delay of $1.7 \mathrm{~ms} /$ eye (95\% CI $0 \cdot 5-2 \cdot 9 ; \mathrm{p}=0 \cdot 0048)$ in the crossover model. The clinical effect observed for group 1 was also sustained into the second epoch (figure 2). Given this sustained effect the so-called no-carryover assumption of the standard crossover model led to a substantial underestimate of the magnitude of the therapeutic effect of clemastine fumarate. Therefore, a delayedtreatment model was also assessed post hoc, showing a $3.2 \mathrm{~ms}$ reduction in VEP latency delay per eye $(1 \cdot 8-4 \cdot 7$; $\mathrm{p}=0 \cdot 0001)$ while on therapy. Furthermore, $16 \%$ of group 1 and $26 \%$ of group 2 showed a latency improvement of more than $6 \mathrm{~ms}$ while on treatment compared with $3 \%$ of group 1 and $6 \%$ of group 2 while on placebo (figure 2) in a post-hoc analysis done based on lab standards of significant interocular differences and previous scientific literature. ${ }^{33,34}$ Patients also showed evidence of improvement in LCLA for the period on treatment, with an increase of 0.9 letters per eye $(95 \% \mathrm{CI}$ -0.1 to $1.9 ; \mathrm{p}=0.085$; figure 3 ) using the crossover analysis; however, this observation did not meet the prespecified threshold of statistical significance. An

\begin{tabular}{|c|c|c|}
\hline & Group $1(n=25)$ & Group $2(n=25)$ \\
\hline Age (years) & $40 \cdot 2(10 \cdot 8)$ & $40 \cdot 0(10 \cdot 1)$ \\
\hline \multicolumn{3}{|l|}{ Sex } \\
\hline Female & $19(76 \%)$ & $13(52 \%)$ \\
\hline Male & $6(24 \%)$ & $12(48 \%)$ \\
\hline Disease duration (years) & $5.7(6.5)$ & $4 \cdot 4(3 \cdot 6)$ \\
\hline EDSS & $2 \cdot 2(1.0)$ & $2 \cdot 1(1 \cdot 2)$ \\
\hline History of ON & $15(60 \%)$ & $13(52 \%)$ \\
\hline Time since ON (years) & $3.7(3.4)$ & $4 \cdot 9(4 \cdot 6)$ \\
\hline VEP P100 latency (ms) & $128 \cdot 6(11 \cdot 6)$ & $126.8(9.4)$ \\
\hline \multicolumn{3}{|l|}{ OCT } \\
\hline $\mathrm{RNFL}, \mu \mathrm{m}$ & $90 \cdot 2(12 \cdot 0)$ & $85 \cdot 1(7 \cdot 9)$ \\
\hline Macular volume $\left(\mathrm{mm}^{3}\right)$ & $3.05(0.14)$ & $3.01(0.11)$ \\
\hline LCLA & $24.0(8 \cdot 4)$ & $21 \cdot 6(10 \cdot 7)$ \\
\hline SDMT & $51 \cdot 8(10 \cdot 2)$ & $50 \cdot 0(11.1)$ \\
\hline MAF & $17 \cdot 82(12 \cdot 39)$ & $20.43(10 \cdot 88)$ \\
\hline 6-min walk (feet) & $1742 \cdot 40(288 \cdot 14)$ & $1741.76(260.08)$ \\
\hline 25-foot walk (s) & $3.81(0.67)$ & $4 \cdot 10(1.01)$ \\
\hline Myelin water fraction & $67.55(11.85)$ & $65.70(12.98)$ \\
\hline \multicolumn{3}{|l|}{ MTR 25} \\
\hline Brain & $0.39(0.05)$ & $0.38(0.03)$ \\
\hline White matter & $0.54(0.02)$ & $0.54(0.02)$ \\
\hline FA white matter & $0.24(0.02)$ & $0.24(0.01)$ \\
\hline \multicolumn{3}{|c|}{$\begin{array}{l}\text { Data are mean (SD) or } \mathrm{n}(\%) \text {. Group } 1 \text { received active treatment (clemastine } \\
\text { fumarate) for } 90 \text { days, followed by placebo for } 60 \text { days. Group } 2 \text { received placebc } \\
\text { for } 90 \text { days, followed by active treatment (clemastine fumarate) for } 60 \text { days. } \\
\text { EDSS=Expanded disability status scale. } O N=\text { optic neuritis. VEP=visual-evoked } \\
\text { potential. OCT=optical coherence tomography. } R N F L=\text { retinal nerve fibre layer } \\
\text { thickness. } L C L A=\text { low-contrast letter acuity. SDMT=Symbol Digit Modality Test. } \\
M A F=\text { multimodal assessment of fatigue. MTR=magnetisation transfer ratio. } \\
F A=\text { fractional anisotropy. }\end{array}$} \\
\hline
\end{tabular}

additional post-hoc analysis done by assessing the LCLA outcome with the delayed-treatment model suggested an increase of 1.6 letters per eye $(0 \cdot 2$ to $3 \cdot 0 ; p=0 \cdot 022)$. No effects of age, sex, disease duration, or previous history of clinical optic neuritis were detected.

Groups at baseline did not differ with regards to any of the non-visual functional or MRI metrics (table). None of the predefined MRI measures, including new and enlarging T2 lesions, volume of gadolinium-enhancing lesions on T1, MWF, MTR, or FA on diffusion tensor imaging showed evidence of improvement or worsening while on study drug (appendix) either using the crossover or delayed-treatment analyses. The two groups did not differ in processing speed on study drug, as assessed by the SDMT. Additionally, EDSS, T25W, and 6MWT were unchanged by treatment with clemastine fumarate (appendix).

Serious adverse events did not occur during the trial. Few adverse events were reported: a modest worsening of fatigue from baseline across both cohorts for the period on treatment versus the period off treatment based on the MAF assessment $(p=0 \cdot 017)$. Given the potential for participant unmasking as a consequence of 


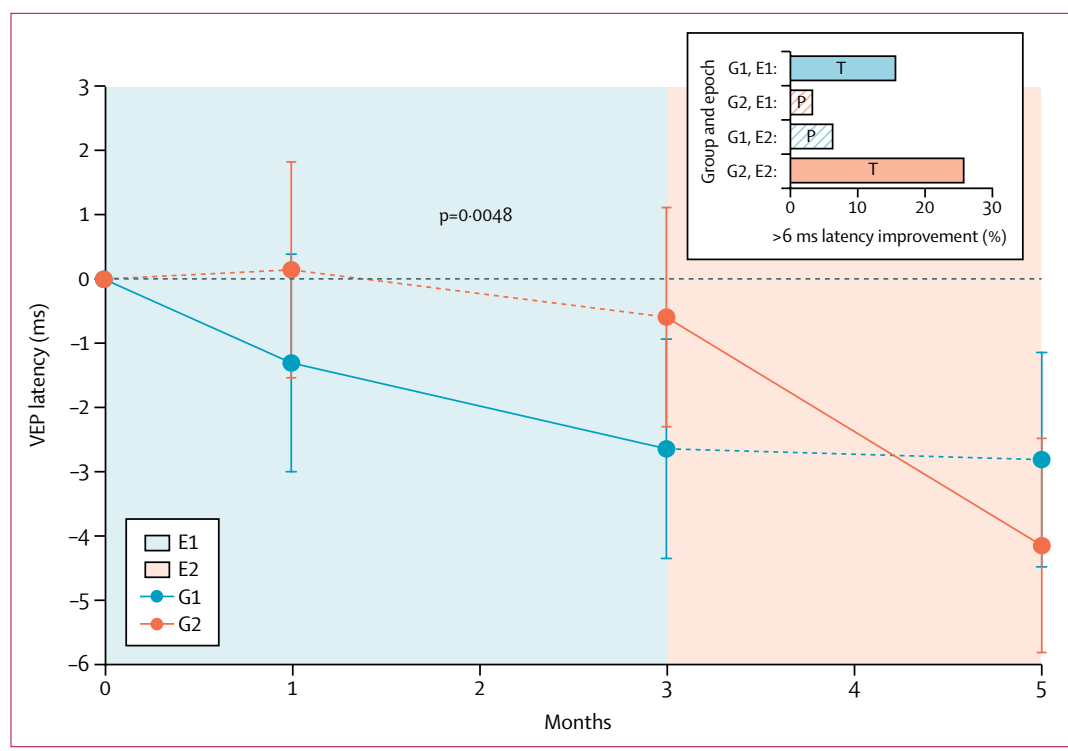

Figure 2: Association of clemastine fumarate treatment with VEP latency delay in patients with chronic optic neuropathy

Change from baseline in latency by group and epoch (model-derived estimates of means are represented by dots with the SE from baseline represented by error bars at each relevant timepoint). Solid line is on-treatment and dashed line is on-placebo. Blue line is group 1, orange line is group 2. Blue shaded area is epoch 1 and orange shaded area is epoch 2. p value is for primary analysis including crossover (with assumption of carryover). The inset is the percentage of patients with more than $6 \mathrm{~ms}$ improvement in latency delay. VEP=visual-evoked potential. G1=group 1. G2=group 2. E1=first epoch. E2=second epoch. $\mathrm{T}$ =treatment period. $\mathrm{P}=$ =placebo period.

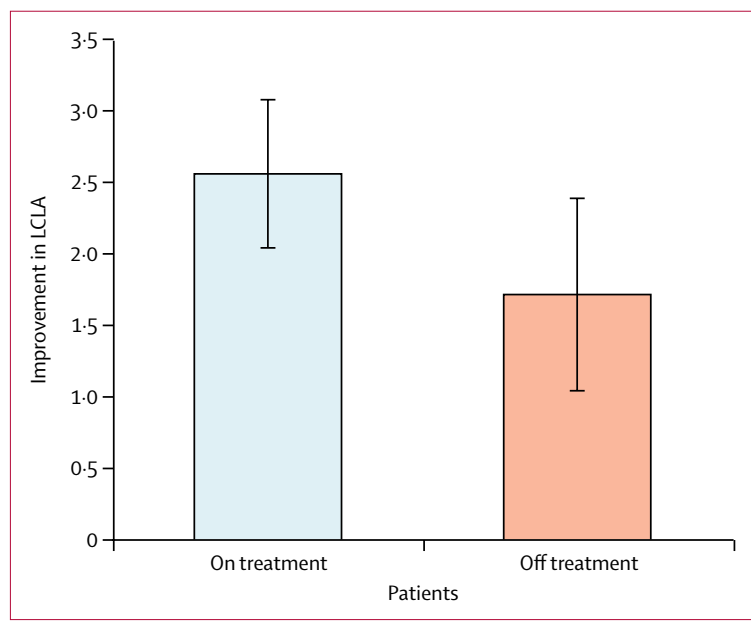

Figure 3: Association of clemastine fumarate treatment with performance on LCLA testing

Change is in number of letters identified correctly. Mean (SD) shown with both epochs combined. $\mathrm{p}=0.085$. LCLA=low-contrast letter acuity.

fatigue, we did additional analyses to determine whether the primary trial result remained significant after controlling for fatigue. Controlling for fatigue had no effect on either the magnitude or statistical significance of the VEP or LCLA results (appendix). One patient had to modify dosing and timing of clemastine fumarate as a result of severe fatigue. Furthermore, a small number of patients exhibited increases in triglyceride concentrations over the course of the trial; however, this outcome had no discernible association to treatment.

\section{Discussion}

To our knowledge, this clinical trial in conjunction with published preclinical evidence related to clemastine fumarate and remyelination provides the first evidence of drug-induced repair in a chronic neurodegenerative condition. Preclinical data unequivocally showed that clemastine fumarate promotes oligodendrocyte precursor differentiation ${ }^{17,18}$ and remyelination ${ }^{19-22}$ without modulating the immune system..$^{19}$ The robustness of the findings were documented by the latency improvement observed in both groups of the cohort while on active treatment. Furthermore, the sustained clinical response in the first epoch provides evidence that the observed improvement was not due to a transient effect of medication on electrical conductance but rather reflects a persistent structural change induced by treatment. Previous studies (NCT02040298) 35 suggested that drugs that target the immune system or paranodal potassium channels might reduce VEP latency.

To show functional repair and regeneration over short timescales in phase 2 clinical trials is challenging in a multifocal, stochastic disease of the CNS, such as multiple sclerosis, because of the wide variety of deficits that can be encountered. The simplification of the phenotype being studied by selecting patients on the basis of shared clinical deficits in an isolated functional pathway allowed for assessment of efficacy using a single clinical outcome. The visual pathway was selected as a suitable model for assessment because of its anatomical segregation and the precision of the clinical tests available for visual assessment. ${ }^{23,26,28}$ The visual system almost universally exhibits injury in people with multiple sclerosis. ${ }^{25,36}$ This trial highlights the value of this model for assessment of remyelination treatments in the chronic disease setting. We included patients who did not have a previous history of optic neuritis. A post-hoc analysis evaluating the history of optic neuritis showed the robustness of the effect of clemastine fumarate (despite the reduction in the size of the stratified sample) but could be taken to suggest that patients with a previous clinical episode of optic neuritis might have had a more pronounced response (appendix).

Optimal trial design and analytical methods for early phase studies to assess drugs with potential for remyelination and repair have not been determinedespecially for over-the-counter medications. The crossover trial design helped to establish the sustainability of the clinical benefit of clemastine fumarate and enhanced the power of the study to detect an effect in a relatively small population. Furthermore, it made recruitment for a trial with a non-prescription medication possible because all patients were granted access to the study drug. However, the standard statistical approach for analysing crossover study data includes an assumption that there is no carryover of treatment effect-ie, any benefit from earlier clemastine fumarate treatment is not sustained during the control epoch. This assumption did not hold in this study, 
which points to the potential reparative effect of the medication. However, the magnitude of the benefit of clemastine fumarate in this model is estimated on the basis of this assumption of no carryover. Thus, a standard crossover analytical approach underestimates the true magnitude of the therapeutic effect because it falsely discounts the sustained benefit in epoch 2 for group 1 . The benefit of clemastine fumarate treatment on VEP latency showed substantial carryover (ie, the improvement in latency plateaus and persists after cessation of treatment which-given the goal of structural repair-is a desirable outcome). Furthermore, greater improvements in latency for group 2 patients than for group 1 patients were seen during their period on treatment; however, given the assumption about no carryover, the primary analysis underestimates the contribution of this effect (as the sustained group 1 benefits are compared with the group 2 benefits on treatment). Therefore, an assessment of the study results as a delayed-treatment trial provides an accurate estimate of the magnitude of the therapeutic effect. We have presented both methods of analysis to show that a treatment effect is observed using either analytical method. However, we consider the delayedtreatment analysis to provide a more realistic appraisal of the magnitude of the therapeutic benefit achieved with clemastine fumarate at this dose. Additionally, in a post-hoc analysis that evaluated the VEP data using a $6 \mathrm{~ms}$ improvement threshold (which, based on experience and previous literature, ${ }^{33,34}$ would not be anticipated to be due to measurement error), clemastine fumarate treatment outperformed placebo in both periods of the trial.

Given the absence of measurable difference in efficacy between the two groups (which had different lengths of exposure to the active compound), we cannot determine the optimal length of exposure to clemastine fumarate or if additional benefits would be seen with longer exposure. In fact, a modestly greater magnitude of effect was observed in group 2, but the significance of this observation cannot be determined because it could be due to baseline differences between the groups that were not balanced by randomisation. In addition, although the sustained clinical response of group 1 indicates that the clinical effect is not transient, whether the clinical response is maintained over the long term (ie, longer than 2 months) is uncertain.

This trial provides a framework for future assessments of remyelinating therapies for patients with multiple sclerosis. In particular, these results indicate that evoked potentials are a promising and sensitive measure of putative remyelination with effective drugs. The trial also supports the potential clinical benefit of adding a remyelinating drug in stable patients on a pre-existing immunomodulatory therapy.

Patients showed evidence of improvement in LCLA performance that did not meet our prespecified standard for statistical significance using the crossover analysis. However, when analysed with the delayed-treatment model, improvements in LCLA reached statistical significance. LCLA is the best-assessed and most well validated functionally relevant psychophysical clinical trial outcome for multiple sclerosis. ${ }^{26,29}$ This clinical effect was confounded by a learning effect observed for LCLA assessments over the course of the trial. This made detection of clinical effects of the drug more difficult. Future investigations might seek to use additional tests of visual function to help further resolve the clinical effect of treatment.

Preselected MRI measures were unable to detect biological effects. In general, these measures exhibited greater variability than is optimal for clinical trial outcomes. Future studies looking at MRI metrics might need to increase the frequency of assessments to reduce variability and enhance the ability to detect a response. Additional evaluation of our MRI results to assess for novel or promising new ways to analyse the data will be the subject of further study.

Patients in the trial showed worsened fatigue on the basis of MAF while on treatment. Fatigue is reported as a symptom in $65-95 \%$ of patients with multiple sclerosis. As many as $40 \%$ of patients report that it is the most disabling symptom of their disease. ${ }^{37}$ We cannot determine if this fatigue effect is due to action on the target muscarinic receptor or might reflect effects on other receptors, such as histamine receptors. Clemastine fumarate is well known to have antagonistic effects on a wide variety of membrane-bound G-protein-coupled neurotransmitter receptors..$^{38}$ Furthermore, animal experiments indicate that at our selected dose we only achieved partial saturation of the target muscarinic receptor (data not shown; dose adjusted). Non-selective drugs with anticholinergic effects have been suggested to have anticognitive effects in uncontrolled studies in patients with multiple sclerosis..$^{39}$ A significant anticognitive effect was not observed in this trial at doses exceeding existing standard recommendations.

A limitation of the current trial is that it cannot ascertain whether the potential remyelinating effects of clemastine fumarate are sustained beyond the period of study, nor can it assess if continued dosing of clemastine fumarate would yield continued benefits. It cannot be used to assess whether higher doses would provide enhanced beneficial effects and it certainly cannot evaluate the risks of such higher or alternative dosing regimens. We also could not establish if the effects seen in the visual system might reflect that this pathway is more amenable to repair than others, nor given the size of the study could we fully evaluate if certain patient characteristics are most likely to be associated with the observed benefits.

The stability of myelin in the adult CNS remains a matter of controversy. ${ }^{40}$ The results reported here suggest the dynamic potential of an endogenous precursor population for medically induced remyelination in adults with multiple sclerosis. Future work will be needed to establish the longer-term effect of remye- 
lination on prevention of axonal loss and disability progression, identify the extent of repair that is achievable with more selective and targeted drugs, and define the optimal dosing regimens and duration of treatment. Additional preclinical work should address the specificity of VEP latency changes in animal models using drugs such as clemastine fumarate for remyelination. Furthermore, we will need to determine optimal timing of treatment by evaluation of other stages of disease, such as following acute demyelinating injury. This work shows that remyelination and repair are promising goals for medical therapeutics in multiple sclerosis.

\section{Contributors}

AJG designed, conceived, and developed the trial, secured funding, oversaw all aspects of data collection analysis, and wrote the manuscript. JMG designed the trial, recruited patients, acquired data, was an evaluating physician, and critically revised the manuscript. BAC designed the trial, recruited patients, acquired data, was a treating clinician, and critically revised the manuscript. CB executed the trial, acquired data, was an evaluating physician, and critically revised the manuscript. WJB wrote the statistical plan, analysed the data, and critically revised the manuscript. FM, JI, SA, AA, HN, AZ, MF, RG, and HCvB acquired data and critically revised the manuscript. MD acquired and analysed data, and critically revised the manuscript. RGH oversaw all MRI data acquisition, analysed the data, and critically revised the manuscript. SLH designed the trial and critically revised the manuscript. JRC designed the trial, analysed the data, and critically revised the manuscript.

\section{Declaration of interests}

JMG reports grants and personal fees from Genentech, grants from Quest Diagnostics and MedImmune, and personal fees from Medical Legal Consulting, outside the submitted work. BAC reports personal fees for consulting from Abbvie, Biogen, EMD Serono, Novartis, Sanofi Genzyme, and Shire, outside the submitted work. HCvB reports that he is adjunct faculty at University of California San Francisco, and since March, 2016, a full-time employee of F Hoffman-La Roche. RGH reports grants from Roche-Genentech and personal fees from Sanofi-Genzyme, Novartis, Abbvie, and Teva, outside the submitted work. SLH serves on the Scientific Advisory Boards for Annexon, Symbiotix, Bionure, and Molecular Stethoscope. He is on the Board of Trustees for Neurona Therapeutics. SLH has also received travel reimbursement and writing assistance from F Hoffman-La Roche for CD20-related meetings and presentations. AJG reports grants and other support from Inception Biosciences, other support from MedImmune, grants from the National MS Society and US National Institutes of Health, other support from Mylan, Sandoz, Dr Reddy, Amneal, Momenta, Synthon,

JAMA Neurology, and Bionure, outside the submitted work. All other authors have no competing interests.

\section{Acknowledgments}

We would like to thank the Rachleff Family for their generous support of this research. We would additionally like to thank David Rowitch of the Eli and Edythe Broad Institute for Stem Cell Research and Regeneration Medicine and Howard Hughes Medical Institute, University of California (San Francisco, CA, USA) and the Department of Pediatrics, Wellcome Trust-MRC Stem Cell Institute, University of Cambridge (Cambridge, UK) for his feedback and assistance with performing the human oligodendrocyte progenitor cell experiments. We would also like to thank Christopher Songster for his technical assistance and longstanding contributions to our electrophysiology laboratory. We also wish to provide profound thanks to our patients for their willingness to participate in research as well as their courage and enthusiasm.

\section{References}

1 Frohman EM, Racke MK, Raine CS. Multiple sclerosis-the plaque and its pathogenesis. $N$ Engl J Med 2006; 354: 942-55.

2 Hauser SL, Goodin DS. Multiple sclerosis and other demyelinating diseases. In: Harrison's Principles of Internal Medicine. http://accessmedicine.mhmedical.com/content.aspx?bookid=331\&s ectionid=40727196 (accessed Jan 4, 2016).
3 Emery B. Regulation of oligodendrocyte differentiation and myelination. Science 2010; 330: 779-82.

4 Miron VE, Kuhlmann T, Antel JP. Cells of the oligodendroglial lineage, myelination, and remyelination. Biochim Biophys Acta 2011; 1812: 184-93.

5 Czopka T, Ffrench-Constant C, Lyons DA. Individual oligodendrocytes have only a few hours in which to generate new myelin sheaths in vivo. Dev Cell 2013; 25: 599-609.

6 Scolding N, Franklin R, Stevens S, et al. Oligodendrocyte progenitors are present in the normal adult human CNS and in the lesions of multiple sclerosis. Brain 1998; 121: 2221-28.

7 Wolswijk G. Chronic stage multiple sclerosis lesions contain a relatively quiescent population of oligodendrocyte precursor cells. J Neurosci 1998; 18: 601-09.

8 Kuhlmann T, Miron V, Cui Q, Wegner C, Antel J, Brück W. Differentiation block of oligodendroglial progenitor cells as a cause for remyelination failure in chronic multiple sclerosis. Brain 2008; 131: 1749-58.

9 Périer O, Grégoire A. Electron microscopic features of multiple sclerosis lesions. Brain 1965; 88: 937-52.

10 Frischer JM, Weigand SD, Guo Y, et al. Clinical and pathological insights into the dynamic nature of the white matter multiple sclerosis plaque. Ann Neurol 2015; 78: 710-21.

11 Hauser SL, Chan JR, Oksenberg JR. Multiple sclerosis: prospects and promise. Ann Neurol 2013; 74: 317-27.

12 Brück W, Gold R, Lund BT, et al. Therapeutic decisions in multiple sclerosis: moving beyond efficacy. JAMA Neurol 2013; 70: $1315-24$

13 Polman CH, Reingold SC, Banwell B, et al. Diagnostic criteria for multiple sclerosis: 2010 revisions to the McDonald criteria. Ann Neurol 2011; 69: 292-302.

14 Lassmann H, Brück W, Lucchinetti CF. The immunopathology of multiple sclerosis: an overview. Brain Pathol 2007; 17: 210-18.

15 Bjartmar C, Trapp BD. Axonal and neuronal degeneration in multiple sclerosis: mechanisms and functional consequences. Curr Opin Neurol 2001; 14: 271-78.

16 Waxman SG. Ions, energy and axonal injury: towards a molecular neurology of multiple sclerosis. Trends Mol Med 2006; 12: 192-95.

17 Mei F, Fancy SP, Shen YA, et al. Micropillar arrays as a high-throughput screening platform for therapeutics in multiple sclerosis. Nat Med 2014; 20: 954-60.

18 Deshmukh VA, Tardif V, Lyssiotis CA, et al. A regenerative approach to the treatment of multiple sclerosis. Nature 2013; 502: 327-32.

19 Mei F, Lehmann-Horn K, Shen YA, et al. Accelerated remyelination during inflammatory demyelination prevents axonal loss and improves functional recovery. Elife 2016; 5: e18246.

20 Liu J, Dupree JL, Gacias M, et al. Clemastine enhances myelination in the prefrontal cortex and rescues behavioral changes in socially isolated mice. J Neurosci 2016; 36: 957-62.

21 Li Z, He Y, Fan S, Sun B. Clemastine rescues behavioral changes and enhances remyelination in the cuprizone mouse model of demyelination. Neurosci Bull 2015; 31: 617-25.

22 Zada D, Tovin A, Lerer-Goldshtein T, Appelbaum L. Pharmacological treatment and BBB-targeted genetic therapy for MCT8-dependent hypomyelination in zebrafish. Dis Model Mech 2016; 9: 1339-48.

23 Green AJ. Visual evoked potentials, electroretinography, and othe diagnostic approaches to the visual system. In: Aminoff MJ, ed. Aminoff's electrodiagnosis in clinical neurology, 6th edn. Philadelphia, PA: Elsevier Saunders, 2012: 477-503.

24 Halliday AM, McDonald WI, Mushin J. Visual evoked response in diagnosis of multiple sclerosis. BMJ 1973; 4: 661-64.

25 Toussaint D, Perier O, Verstappen A, et al. Clinicopathological study of the visual pathways, eyes, and cerebral hemispheres in 32 cases of disseminated sclerosis. J Clin Neuroophthalmol 1983; 3: 211-20.

26 Toosy AT, Mason DF, Miller DH. Optic neuritis. Lancet Neurol 2014; 13: 83-99.

27 Horton L, Conger A, Conger D, et al. Effect of 4-aminopyridine on vision in multiple sclerosis patients with optic neuropathy. Neurology 2013; 80: 1862-66.

28 Balcer LJ, Frohman EM. Evaluating loss of visual function in multiple sclerosis as measured by low-contrast letter acuity. Neurology 2010; 74 (suppl 3): S16-23. 
29 Parmenter BA, Weinstock-Guttman B, Garg N, Munschauer F, Benedict RH. Screening for cognitive impairment in multiple sclerosis using the Symbol digit Modalities Test. Mult Scler 2007; 13: 52-57.

30 Ray PG, Meador KJ, Loring DW, et al. Central anticholinergic hypersensitivity in aging. J Geriatr Psychiatry Neurol 1992; 5: 72-77.

31 Dittner AJ, Wessely SC, Brown RG. The assessment of fatigue: a practical guide for clinicians and researchers. J Psychosom Res 2004; 56: $157-70$.

32 Schwartz CE, Coulthard-Morris L, Zeng Q. Psychosocial correlates of fatigue in multiple sclerosis. Arch Phys Med Rehabil 1996; 77: 165-70.

33 Walsh JC, Garrick R, Cameron J, McLeod JG. Evoked potential changes in clinically definite multiple sclerosis: a two year follow up study. J Neurol Neurosurg Psychiatry 1982; 45: 494-500.

34 Niklas A, Sebraoui H, Hess E, Wagner A, Then Bergh F. Outcome measures for trials of remyelinating agents in multiple sclerosis: retrospective longitudinal analysis of visual evoked potential latency. Mult Scler 2009; 15: 68-74.

35 Nuwer MR, Packwood JW, Myers LW, et al. Evoked potentials predict the clinical changes in a multiple sclerosis drug study. Neurology 1987; 37: 1754-61.
36 Green AJ, McQuaid S, Hauser SL, Allen IV, Lyness R. Ocular pathology in multiple sclerosis: retinal atrophy and inflammation irrespective of disease duration. Brain 2010; 133: 1591-601.

37 Hadjimichael O, Vollmer T, Oleen-Burkey M. Fatigue characteristics in multiple sclerosis: the North American Research Committee on Multiple Sclerosis (NARCOMS) survey. Health Qual Life Outcomes 2008; 6: 100.

38 Kubo N, Shirakawa O, Kuno T, et al. Antimuscarinic effects of antihistamines: quantitative evaluation by receptor-binding assay. Jpn J Pharmacol 1987; 43: 277-82.

39 Cruce R, Vosoughi R, Freedman MS. Cognitive impact of anticholinergic medication in MS: Adding insult to injury? Mult Scler Relat Disord 2012; 1: 156-61.

40 Yeung MS, Zdunek S, Bergmann O, et al. Dynamics of oligodendrocyte generation and myelination in the human brain. Cell 2014; 159: 766-74. 


\section{THE LANCET}

\section{Supplementary appendix}

This appendix formed part of the original submission and has been peer reviewed. We post it as supplied by the authors.

Supplement to: Green AJ, Gelfand JM, Cree BA, et al. Clemastine fumarate as a remyelinating therapy for multiple sclerosis (ReBUILD): a randomised, controlled, double-blind, crossover trial. Lancet 2017; published online Oct 10. http://dx.doi.org/10.1016/S0140-6736(17)32346-2. 


\section{Supplementary Methods, Tables and Figure:}

iPSC generation and culture: Human iPSCs were generated from postnatal patient fibroblasts obtained under approval from UCSF Institutional Review Board (IRB). iPSC clones were established by electroporation of a CoMiP episomal vector containing 4 reprogramming factors (Oct4, KIf4, Sox2, and c-Myc) following a published protocol. ${ }^{\mathrm{S} 1}$ The patient carried a mutation, which was corrected to wildtype sequence by gene targeting. iPSCs were maintained on CF1 MEF feeder cells in ES medium containing Knockout DMEM/F12, 20\% knockout serum replacement, non-essential amino acids, Glutamax (Thermo Fisher), and $10 \mathrm{ng} / \mathrm{ml}$ human basic FGF (Peprotech). Pluripotency was confirmed by immunocytochemical staining of Tra1-60 (Cell Signaling) and Oct3/4 (Santa Cruz Biotechnology), as well as teratoma formation and differentiation into ectoderm, mesoderm, and endoderm lineages. A normal karyotype was confirmed.

OPC differentiation from iPSCs and clemastine treatment: A previously published protocol for directed iPSC differentiation to OPCs was used with the following modifications ${ }^{\text {s2-S3 }}$ human ES medium and human ES medium without basic FGF were used in place of mTeSR and custom mTeSR, respectively. The concentration of SAG was $0.5 \mathrm{uM}$, T3 $40 \mathrm{ng} / \mathrm{ml}$, and NT3 1 ng/ml. Penicillin-Streptomycin was omitted from N2 medium, HGF from PDGF medium, and HEPES from Glia medium. On day 0 , iPSCs were plated at $0.25 \times 10^{6}$ cells/well in a matrigelcoated 6-well plate with ES medium without basic FGF, supplemented with dual SMAD inhibitors, RA, and ROCK inhibitor thiazovivin (Santa Cruz). From day 1 to day 4, N2 medium was gradually increased by $25 \%$ each day, reaching $100 \%$ on day 4 . On day 8 , dual SMAD inhibitors were replaced with SAG. On day 12 , cells were lifted, dissociated, and seeded onto petri dishes for sphere formation. On day 20, the medium was changed to PDGF-containing medium. On day 30, spheres were plated on poly-L-ornithine/laminin-coated dishes. On day 45, 
medium was changed to Glial medium to initiate differentiation. Cells were exposed to clemastine at $500 \mathrm{nM}$ from day 45 and analysed on day 55.

Detailed description of MRI protocol: Standard high-resolution T1-weighted acquisition of the brain (sagittal 3D MPRAGE, $1 \mathrm{~mm}^{3}$ cubic voxel, iPAT 2, acquisition time: 5:12 min) and a sagittal 3D FLAIR (1 $\mathrm{mm}^{3}$ cubic voxel, iPAT 2, acquisition time: 6:12 min) were acquired for all participants.

A Magnetization Transfer Ratio (MTR) protocol with correction for RF inhomogeneity and T1 relaxation (MTsat) was also acquired for the brain. The protocol consisted of 2 axial volumes acquired with a 3D FLASH sequence with and without a MT pulse (1.2 kHz offset frequency) applied (resolution $1 \times 1 \times 2.5 \mathrm{~mm}^{3}$, FOV $240 \times 240 \times 180 \mathrm{~mm}^{3}$ ); other key parameters: TR/TE = 29/4.92 ms, flip angle $=5^{\circ}$. A third volume to correct for RF inhomogeneity and T1 relaxation effects was also acquired with a flip angle $=15^{\circ}$. Total acquisition time was about 10:20 min. MTsat values were computed using the methods described by Helms et al. ${ }^{\mathrm{S}}$

The brain Myelin Water Fraction (MWF) protocol consisted of a volume acquired using an axial 2D multi-gradient-echo FLASH sequence (in plane resolution $1.25 \times 1.25 \mathrm{~mm}^{2}$, FOV $240 \times 240$ $\mathrm{mm}^{2}, 50$ slices of $2.5 \mathrm{~mm}$ thickness) with a flip angle of $40^{\circ}, \mathrm{TR}=2260 \mathrm{~ms}$ and total acquisition time of 4:15 $\mathrm{min}$. For each acquisition, 10 echoes were collected with first echo time $\mathrm{TE}_{1}=4.70$ $\mathrm{ms}$ and echo spacing $\Delta \mathrm{TE}=4 \mathrm{~ms}$. To compute $\mathrm{T} 1$ relaxation times and correct for signal saturation effects, a second axial 2D FLASH volume was acquired (TR=700 ms, two echoes with TE 4.70 and $8.70 \mathrm{~ms}$, flip angle $70^{\circ}$ ). A series of low resolution GE-EPI were also acquired to correct for $\mathrm{B}_{0}$ and $\mathrm{B}_{1} \mathrm{RF}$ inhomogeneity. The MWF protocol had a total duration time of about 9 min. Myelin water fractions were computed using the method described by Neeb et al. ${ }^{\text {s5-s6 }}$ 
A two-shell NODDI protocol ${ }^{\mathrm{S7}}$ of total scan time of about 9 min was implemented using an axial multi band SE-EPI sequence with slice acceleration factor 2. Other key acquisition parameters: $\mathrm{TR} / \mathrm{TE}=4300 / 96 \mathrm{~ms}, 2.2 \mathrm{~mm}^{3}$ cubic voxel, 66 axial slices, iPAT 2, 30 directions at $\mathrm{b}=700$ $\mathrm{s} / \mathrm{mm}^{2}, 64$ directions at $\mathrm{b}=2000 \mathrm{~s} / \mathrm{mm}^{2}$ and 10 volumes without diffusion sensitising gradients applied (b0). The diffusion MRI data were processed including eddy current corrections and diagonalization to get parameters of the diffusion tensor model. The fractional anisotropy was calculated from the diffusion tensor eigenvalues (DiPy). 
Supplementary Table 1: Sensitivity Analyses- Table showing results from all different analytical approaches to evaluate robustness of trial results. This includes sensitivity analyses (1-3) ignoring the data from the 1-month assessment, (4) evaluating the trial as a parallelgroups double-blind placebo-controlled trial in epoch 1, (5) controlling for fatigue at each study visit and (6) controlling for history of optic neuritis.

\begin{tabular}{|l|l|l|l|l|}
\hline Model & $\begin{array}{l}\text { Estimated } \\
\text { Treatment } \\
\text { Effect }\end{array}$ & LCL & UCL & p-value \\
\hline $\begin{array}{l}\text { Primary from Protocol } \\
\text { (Crossover with all times) }\end{array}$ & 1.7 & 0.5 & 2.9 & 0.005 \\
\hline $\begin{array}{l}\text { Primary if 1 month data is } \\
\text { ignored }\end{array}$ & 1.9 & 0.4 & 3.4 & 0.014 \\
\hline $\begin{array}{l}\text { Parallel Groups (ignore } \\
\text { month 5) }\end{array}$ & 1.5 & 0.1 & 3.1 & 0.048 \\
\hline $\begin{array}{l}\text { Parallel Groups (ignore } \\
\text { month 1 and month 5) }\end{array}$ & 1.9 & 0.3 & 3.5 & 0.023 \\
\hline $\begin{array}{l}\text { Delayed Treatment } \\
\text { (consider Group 1 as } \\
\text { treatment at 5 month) }\end{array}$ & 3.2 & 1.8 & 4.6 & $<0.0001$ \\
\hline $\begin{array}{l}\text { Primary if controlling for } \\
\text { fatigue (MAF) }\end{array}$ & 1.7 & 0.5 & 2.9 & 0.007 \\
\hline $\begin{array}{l}\text { Primary if controlling for } \\
\text { history of optic neuritis }\end{array}$ & 1.7 & 0.5 & 2.9 & 0.005 \\
\hline $\begin{array}{l}\text { LCLA if controlling for } \\
\text { fatigue crossover }\end{array}$ & 0.91 & -0.18 & 2.0 & 0.101 \\
\hline $\begin{array}{l}\text { LCLA if controlling for } \\
\text { fatigue delayed treatment }\end{array}$ & 1.62 & 0.19 & 3.1 & 0.026 \\
\hline
\end{tabular}


Supplementary Table 2: Secondary Outcomes: Additional outcomes from the clinical trial. All data was analyzed using the standard crossover model specified in the analysis plan in supplementary table $2 \mathrm{a}$ and as a delayed treatment trial in supplementary table $2 \mathrm{~b}$. Effect is therefore the measurement on drug compared to baseline or screening (as appropriate). Sixminute walk test reflects the distance in feet participants walked in 6 minutes. The timed 25-foot walk represents the time taken to walk 25 feet. MTR = Magnetization Transfer Ratio, MWF= Myelin Water Fraction, SDMT= Symbol Digit Modality Test, EDSS= Expanded Disability Scoring System.

a. Crossover model

\begin{tabular}{|l|r|r|}
\hline Outcome & Effect & p-value \\
\hline 6 minute walk (feet) & -14.81 & 0.515 \\
\hline 25 foot walk (s) & 0.04 & 0.601 \\
\hline MTR whole brain & 0.001 & 0.683 \\
\hline MTR White Matter & 0.0004 & 0.86 \\
\hline $\begin{array}{l}\text { Fractional Anisotropy } \\
\text { White Matter }\end{array}$ & 0.0013 & 0.154 \\
\hline MWF whole brain & 0.469 & 0.808 \\
\hline SDMT & -1.714 & 0.109 \\
\hline EDSS & -0.064 & 0.471 \\
\hline
\end{tabular}

b. Delayed treatment model

\begin{tabular}{|l|r|r|}
\hline Factor & Effect & p-value \\
\hline 6 minute walk (feet) & -2.97 & 0.921 \\
\hline 25 foot walk (s) & -0.01 & 0.910 \\
\hline MTR whole brain & -0.003 & 0.310 \\
\hline MTR White Matter & 0.0003 & 0.919 \\
\hline $\begin{array}{l}\text { Fractional Anisotropy } \\
\text { White Matter }\end{array}$ & 0.0009 & 0.452 \\
\hline MWF whole brain & 1.260 & 0.588 \\
\hline SDMT & 0.501 & 0.721 \\
\hline EDSS & -0.019 & 0.868 \\
\hline
\end{tabular}




\section{Supplementary References:}

1. Diecke S, Lu J, Lee J, Termglinchan V, Kooreman NG, Burridge PW, Ebert AD, Churko JM, Sharma A, Kay MA, Wu JC. Novel codon-optimized mini-intronic plasmid for efficient, inexpensive, and xeno-free induction of pluripotency. Sci Rep. 2015 Jan 28;5:8081. doi: 10.1038/srep08081. PubMed PMID: 25628230; PubMed Central PMCID: PMC4308704.

2. Douvaras P, Wang J, Zimmer M, Hanchuk S, O'Bara MA, Sadiq S, Sim FJ, Goldman J, Fossati V. Efficient generation of myelinating oligodendrocytes from primary progressive multiple sclerosis patients by induced pluripotent stem cells. Stem Cell Reports. 2014 Aug 12;3(2):250-9. doi: 10.1016/j.stemcr.2014.06.012. PubMed PMID: 25254339; PubMed Central PMCID: PMC4176529.

3. Mei F, Mayoral S, Nobuta $\mathrm{H}$, et al. Identification of the kappa-opioid receptor as a therapeutic target for oligodendrocyte remyelination. J Neurosci 2016; 36(30): 7925-7935.

4. Helms G, Dathe $H$, Kallenberg K, Dechent $P$. High-resolution maps of magnetization transfer with inherent correction for RF inhomogeneity and T1 relaxation obtained from 3D FLASH MRI. Magn Reson Med 2008; 60(6): 1396-407.

5. Neeb H, Ermer V, Stocker T, Shah NJ. Fast quantitative mapping of absolute water content with full brain coverage. Neuroimage 2008; 42(3): 1094-109.

6. Tonkova, V., Arhelger, V., Schenk, J., \& Neeb, H. (2011). Rapid Myelin Water Content Mapping on Clinical MR Systems. Zeitschrift Fur Medizinische Physik. http://doi.org/10.1016/j.zemedi.2011.09.005

7. Zhang $H$, Schneider T, Wheeler-Kingshott CA, Alexander DC. NODDI: practical in vivo neurite orientation dispersion and density imaging of the human brain. Neuroimage 2012; 61(4): 1000-16. 


\section{Supplementary Figure 1}

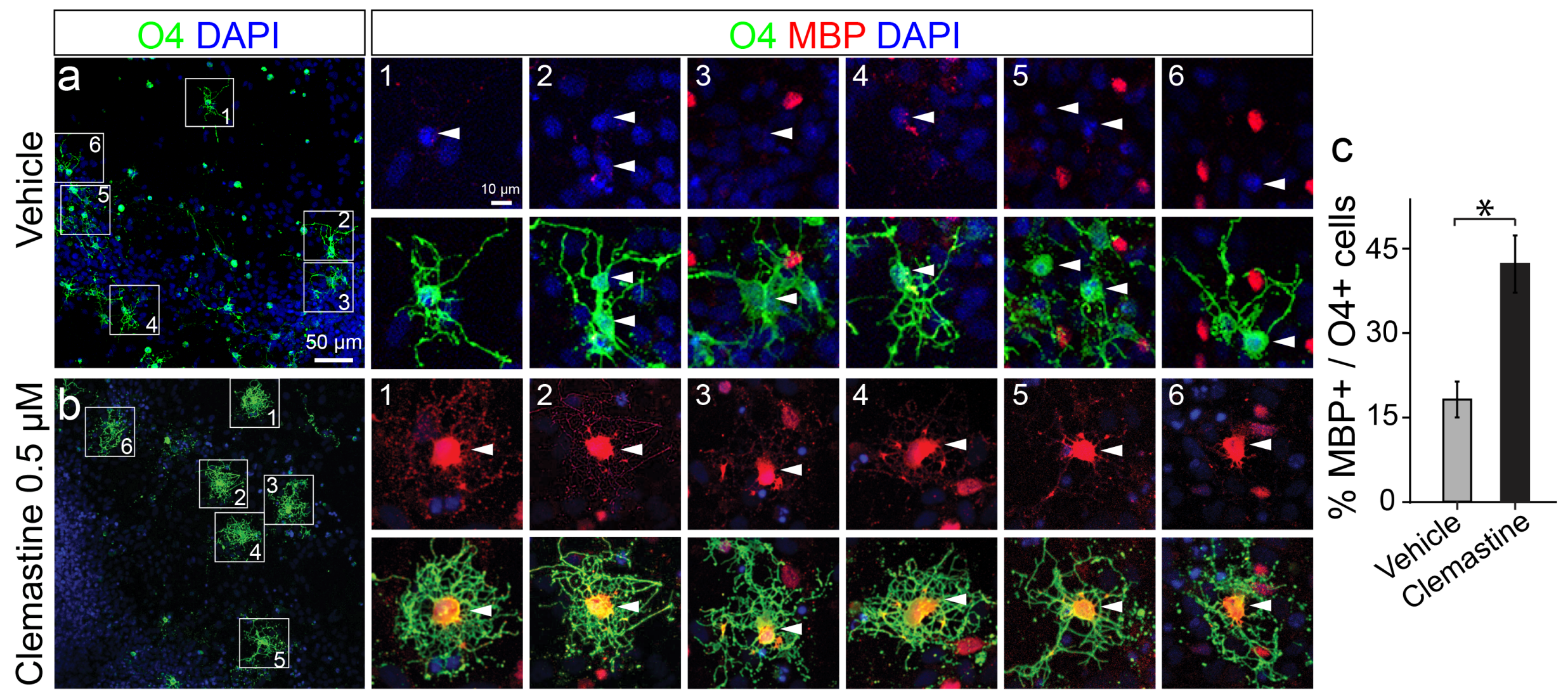

Figure 1. Clemastine enhances the maturation of human iPSC-derived oligodendroglia. Human iPSC-derived oligodendroglia were treated with vehicle or clemastine at $0.5 \mu \mathrm{M}$ for 10 days and immunostained with markers for immature oligodendrocytes (O4; green), differentiated oligodendrocytes (MBP; red), and cell nuclei (DAPI; blue). (a) Representative fields of human iPSC-derived oligodendroglia showing faint MBP immunostaining (red) in vehicle-treated cells while clemastine-treated cells show intense MBP immunostaining and elaborate MBP+ membranes (red) (b). (c) Quantification of the percentage of O4+ cells that are MBP+. Error bars represent mean \pm s.e.m. ${ }^{*} \mathrm{P}<0 \cdot 05$, significance based on Student's t-test. $\mathrm{N}=3$ for all experiments. 